\title{
Bone-Mounted Miniature Robot for Surgical Procedures: Concept and Clinical Applications
}

\author{
Moshe Shoham, Member, IEEE, Michael Burman, Eli Zehavi, Leo Joskowicz, Senior Member, IEEE, Eduard Batkilin,
} and Yigal Kunicher

\begin{abstract}
This paper presents a new approach to robot-assisted spine and trauma surgery in which a miniature robot is directly mounted on the patient's bony structure near the surgical site. The robot is designed to operate in a semiactive mode to precisely position and orient a drill or a needle in various surgical procedures. Since the robot forms a single rigid body with the anatomy, there is no need for immobilization or motion tracking, which greatly enhances and simplifies the robot's registration to the target anatomy. To demonstrate this concept, we developed the MiniAture Robot for Surgical procedures (MARS), a cylindrical $5 \times 7 \mathrm{~cm}^{3}, 200-\mathrm{g}$, six-degree-of-freedom parallel manipulator. We are currently developing two clinical applications to demonstrate the concept: 1 ) surgical tools guiding for spinal pedicle screws placement; and 2) drill guiding for distal locking screws in intramedullary nailing. In both cases, a tool guide attached to the robot is positioned at a planned location with a few intraoperative fluoroscopic X-ray images. Preliminary in-vitro experiments demonstrate the feasibility of this concept.
\end{abstract}

Index Terms-Image-based robot registration and targeting, medical robotics, orthopaedics, surgical robots.

\section{INTRODUCTION}

$\mathbf{R}$ OBOT-ASSISTED surgery (RAS) is an emerging interdisciplinary field whose aim is to provide surgeons with tools that enhance and complement their free-hand abilities during surgery. The goals are to improve the outcome of surgical procedures, to reduce intraoperative time, to reduce the invasiveness of a procedure, or to enable new procedures altogether. Since their inception in the early 1990s, a few dozen surgical robot prototypes have been developed, with the most prominent being the commercial system ROBODOC (Integrated Surgical Systems) and more recently the Da Vinci (Intuitive Surgical) and Zeus (Computer Motion) systems for remotely manipulated minimally invasive procedures. For recent surveys in medical robotics, see [6] and Troccaz et al., 2002.

The main expected advantages of surgical robots are:

- higher accuracy;

Manuscript received February 24, 2003. This paper was recommended for publication by Associate Editor P. Dario and Editor R. Taylor upon evaluation of the reviewers' comments.

M. Shoham is with the Faculty of Mechanical Engineering, Technion-Israel Institute of Technology, Haifa 32000, Israel (e-mail: shoham@tx.technion.ac.il).

M. Burman, E. Zehavi, E. Batkilin, and Y. Kunicher are with Mazor Surgical Technologies, Caesarea 38900, Israel.

L. Joskowicz is with the School of Computer Science and Engineering, The Hebrew University of Jerusalem, Israel (e-mail: josko@cs.huji.ac.il).

Digital Object Identifier 10.1109/TRA.2003.817075
- ability to work according to preplanned image-based program;

- reduction of the surgeon's hand tremor ([18], [26]);

- ability to operate in remotely manipulated minimally invasive procedures;

- reduction of the surgeon and operating staff radiation exposure ([20], [25]);

- reduction of operating room staff.

Despite this extensive and promising list of advantages, and of more than ten years of RAS research, the impact of surgical robots has been very limited, so far, to remotely manipulated minimally invasive procedures. The total worldwide number of surgical robots is less than 1000 . While it is common knowledge that the medical profession is conservative and slow in its adoption of new developments, it appears that the potential of surgical robotics should have resulted in more than a handful of applications.

There are several reasons for the slow assimilation of surgical robots in the operating room. We focus in particular on three main limitations of surgical robots.

- Contemporary medical robots are voluminous. They occupy too much precious operating room space and raise safety issues.

- Commercial surgical robot systems are expensive ( $\$ 300000$ to $\$ 1000000$ ). Their use is thus limited to the few large research hospitals that can afford them.

- The patient anatomy needs to be immobilized by fixing it to the operating room table, or compensated for by tracking it in real time and adjusting the fixed robot position accordingly.

In this paper, we propose a new approach to medical robotics that addresses these issues with a miniature robot that is directly mounted on the patient anatomy. Table I summarizes the characteristics of this type of system as compared to navigation and fixed floor- or bed-mounted robots. We first describe the concept, an embodiment of it, and discuss two clinical applications currently under development: 1) surgical tool guiding for spinal pedicle screws placement; and 2) drill guiding for distal locking screws in intramedullary nailing.

\section{Bone-Mounted Miniature Robot: RATIONALE AND SYSTEM CONCEPT}

Many minimally invasive clinical applications for which RAS is applicable require a small operating workspace. Examples include spine surgery, femoral head fracture fixation, cardiac 
TABLE I

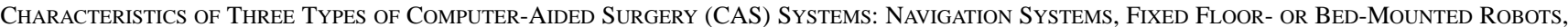
AND PATIENT-MOUNTED RoBOTS

\begin{tabular}{|l|l|l|l|l|}
\hline $\begin{array}{l}\text { CAS syste m } \\
\text { type }\end{array}$ & \multicolumn{1}{|c|}{ Accuracy } & $\begin{array}{l}\text { Operating Room } \\
\text { space }\end{array}$ & \multicolumn{1}{c|}{ Simplicity } & Safety \\
\hline $\begin{array}{l}\text { Navigation } \\
\text { systems }\end{array}$ & $\begin{array}{l}\text { Accuracy is limited by the } \\
\text { surgeon free hand motion }\end{array}$ & $\begin{array}{l}\text { Requires a tracking } \\
\text { system with either } \\
\text { direct line of sight } \\
\text { or non-ferrous } \\
\text { instruments. }\end{array}$ & $\begin{array}{l}\text { Requires manual } \\
\text { spatial tool } \\
\text { alignment based on } \\
\text { screen image and a } \\
\text { setup for direct line } \\
\text { of sight }\end{array}$ & $\begin{array}{l}\text { All tools are marked. No } \\
\text { mechanical arm. Relies on a } \\
\text { real-time tracking system. }\end{array}$ \\
\hline $\begin{array}{l}\text { Floor/bed } \\
\text { mounted } \\
\text { surgical } \\
\text { robot }\end{array}$ & $\begin{array}{l}\text { Medium relative accuracy } \\
\text { due to the robot remote } \\
\text { location and require to } \\
\text { either immobilize or to } \\
\text { track anatomy motion in } \\
\text { real-time }\end{array}$ & $\begin{array}{l}\text { Requires large } \\
\text { space around the } \\
\text { operating room } \\
\text { table }\end{array}$ & $\begin{array}{l}\text { Requires patient } \\
\text { immobilization or } \\
\text { real-time tracking }\end{array}$ & $\begin{array}{l}\text { Large robot motion and high } \\
\text { inertia potentially unsafe to } \\
\text { the patient and staff. }\end{array}$ \\
\hline $\begin{array}{l}\text { Patient } \\
\text { mounted } \\
\text { surgical } \\
\text { robot }\end{array}$ & $\begin{array}{l}\text { High accuracy due to the } \\
\text { robot structure and the } \\
\text { close proximity to the } \\
\text { surgical site }\end{array}$ & $\begin{array}{l}\text { Requires minimal } \\
\text { operating room } \\
\text { space }\end{array}$ & $\begin{array}{l}\text { No need for patient } \\
\text { immobilization or } \\
\text { patient attached } \\
\text { dynamic } \\
\text { referencing. }\end{array}$ & $\begin{array}{l}\text { Small workspace in the } \\
\text { vicinity of the surgical site. } \\
\text { Low inertia and small motors } \\
\text { that cannot harm the patient } \\
\text { or the operating room staff. }\end{array}$ \\
\hline
\end{tabular}

procedures, prostate procedures, ear, nose, and throat (ENT) surgery, dental surgery, neurosurgery, and a variety of biopsies. The workspace required for positioning and operating the surgical tools, e.g., needles and drills, is enclosed in a sphere whose radius is only several centimeters.

Realizing that the robot workspace is small has important consequences on the robot design and use. First, it justifies designing a robot that is not much larger than its workspace. Second, small robots are intrinsically safer than large robots. Third, small robots can be directly mounted on the patient anatomy, usually a bony structure near the surgical site. Because it is attached to the patient, there is no need for immobilization or motion tracking, which greatly simplifies the robot's registration to the target anatomy and its accuracy.

Having determined the desired robot characteristics and work volume, the next step consists of choosing a suitable robot architecture. Parallel robots, which usually have small work volume, compact design, high accuracy, and high payload-to-weight ratio offer many advantages over common serial robots. In fact, some recent investigations have shown the potential of parallel robot architectures in medical applications [3], [21], [22], [24], [31].

Based on these considerations, we have developed a miniature surgical robot with a parallel architecture that can be directly mounted on the bone. The robot has attached to its moving platform a surgical instrument or tool guide that can be precisely positioned and oriented to a desired location close to the mounting site. The miniature robot is designed to be the central component of a family of RAS systems for specific clinical applications requiring precise and steady positioning of surgical instruments.

We envisage the following use of a miniature bone-mounted robot in a RAS system whose purpose is minimally invasive guiding and targeting of needles, drills, and other surgical tools. It consists of five steps: 1) preoperative planning, in which the surgeon plans the desired orientation, entry point, and depth of one or more drill or needle procedures based on X-ray, computer tomography (CT), or magnetic resonance imaging (MRI) images; 2) intraoperative robot attachment, in which the sterilized robot with the targeting guide is rigidly attached with a minimally invasive attachment jig to the bony structure close to the surgical site; 3 ) robot registration, in which a precise geometric relation between the coordinate systems of the robot, the target anatomy, and the plan is established; 4) robot positioning, in which the robot controller moves the targeting guide to its planned position and locks the robot in place; and 5) manual execution, in which the surgeon executes drilling or needle insertion through the positioned guide. Steps 4) and 5) are repeated for each planned location.

Robot registration is the key step that determines the RAS system spatial accuracy (besides the intrinsic mechanical positioning errors of the robot, which are usually much smaller). We propose to use fluoroscopic X-ray images, which are routinely used in many surgical procedures, for robot registration. Fluoroscopic X-rays well provide bony structures, and their field of view includes the entire robot working volume. When more than one image is acquired from different viewpoints, each must be corrected for orientation-dependent distortion [2], [13], [32].

\section{EXAmple of A Bone-Mounted Miniature ROBOT: THE MARS ROBOT}

For the type of minimally invasive surgeries under consideration, we identified the following design goals for the miniature bone-mounted robot:

- precise position and orientation of long, handheld surgical instruments, such as a drill or a needle, with respect to a surgical target;

- small work volume enclosing a sphere whose radius is several centimeters;

- rigid attachment to the bone;

- lightweight and compact structure; 


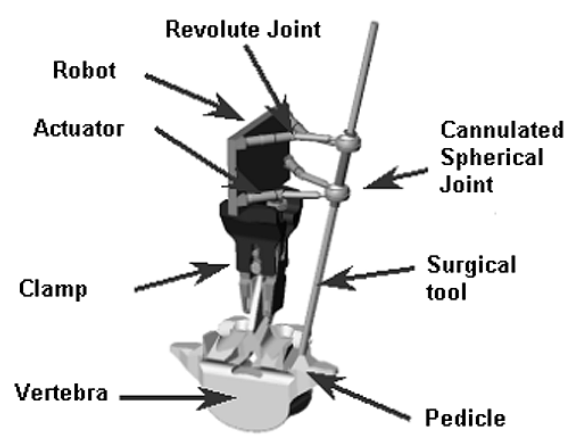

Fig. 1. A four DOF parallel bone-mounted robot concept. The robot is mounted on the spinous process with a clamp.

- lockable structure at given configurations to provide rigid guidance;

- capable of withstanding lateral forces resulting from instrument guidance of up to $10 \mathrm{~N}$;

- modular design to allow customization of the bone attachment and targeting guide for different surgical applications;

- repeatedly sterilizable in its entirety or easily covered with a sterile sleeve;

- quick and easy installation and removal from the bone.

We chose a parallel robot structure to achieve these goals, since this structure has several advantages over serial structures in terms of accuracy, rigidity, and especially compactness. Since the goal of the robot is to position an instrument tip and orient its axis (equivalent to positioning a line in space), a kinematic structure with at least four degrees of freedom (DOFs) is required. Fig. 1 shows one such structure. Note that in some cases, it is necessary to also limit the insertion depth of the needle or drill. This can be achieved by adding a mechanical stop at a predefined location on the drill or needle, or by adding a fifth DOF. We finally settled on a parallel structure with six DOFs. The additional DOF can be used to avoid singular workspace regions and forbidden anatomical configurations.

The next step consisted of determining the desired robot work volume and force characteristics. We conducted a detailed study for pedicle screw insertion in spinal fusion (for a complete report, see [28]). The same robot can be used in other spinal operations where reaching a precise location in the vertebra is required, such as vertebroplasty, discography, and various biopsies.

Based on these considerations, we developed the MiniAture Robot for Surgery (MARS) shown in Fig. 2. MARS is a miniature parallel structure with six DOFs that is directly mounted on the patient anatomy, usually a bony structure near the surgical site. It consists of a fixed base that attaches to the bone and a moving platform connected in parallel by six independent linear actuators. The robot dimensions are $5 \times 5 \times 7 \mathrm{~cm}^{3}$, its weight is $200 \mathrm{~g}$, and its work volume is contained in a sphere whose radius is several centimeters, which is sufficient for a number of surgical procedures. A three-dimensional view of its work volume is shown in Fig. 3. The solution of the inverse and forward kinematics of Gough-Stewart parallel robot is known, see, for example, [15] and [30]. Its positional accuracy is better than 0.1

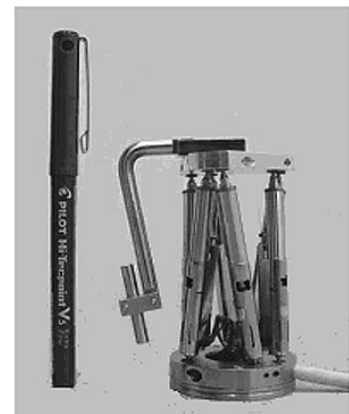

Fig. 2. Photograph of the MARS. The robot is shown with an angled drill guide mounted on its upper base.

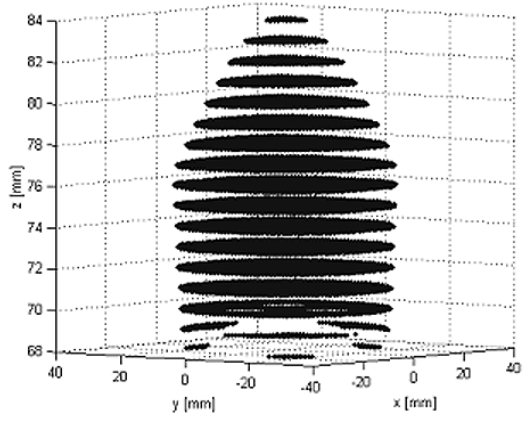

Fig. 3. Work volume of the MARS Robot. Each slice shows the translational $X Y$ range for a given $Z$ value.

$\mathrm{mm}$, which is by far sufficient for most clinical applications and better than the accuracy of commercial tracking systems.

The robot is designed to operate in a semiactive mode, that is, to position and orient the targeting guide to a precise preoperatively defined location and lock itself there, but not to actually perform the surgical operation itself. It provides a mechanical guide for a surgical tool operated by the surgeon. The robot comes with a PC-based controller card that receives joint position feedback and calculates the inverse kinematics for jointlevel control.

\section{Pedicle Screw InSERTion In Spinal Fusion}

The first clinical application of the bone-mounted miniature robot concept is pedicle screw insertion in spinal fusion. Indications for spinal fusion with pedicle screw fixation include: fracture of vertebral body, degenerative disc disease (disc herniation, instability of facet joint, compressive radiculopathy), spine tumors, and scoliosis. The technique calls for disabling the relative motion between adjacent vertebras to prevent compression during body movements and stabilizing the spinal column. Spinal fusion is very common, with over 400000 procedures performed annually in the USA alone. The procedure is performed throughout the spine, on lower lumbar vertebra, middle thoracic vertebra, and on upper cervical vertebra.

The procedure involves inserting two screws per fused vertebra, on the left and right spinal pedicles (Fig. 4). The pedicle screws are inserted at an angle so as to avoid the perforation of the pedicle and damage to the spinal cord or the roots. Once in place, the pedicle screws are fitted with a screw head with a hole into which a rigid rod is inserted. The rod connects the pedicle screw heads on each side, thus forming a single rigid body with 


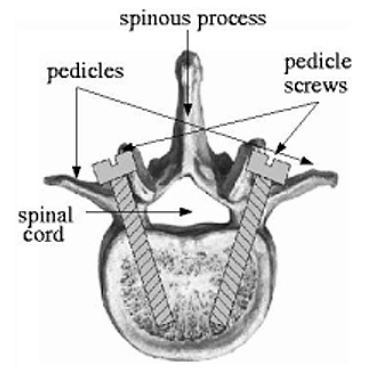

Fig. 4. Diagram showing an axial view of a vertebra with two pedicle screws inserted.

no internal relative motions. The surgery calls for surgically exposing the vertebral pedicles, drilling a pilot hole for each screw, and inserting the screw, all under fluoroscopic $\mathrm{X}$-ray guidance. The pedicle screw heads are then fitted, the rods are inserted, and the surgical wound is closed.

A difficult, error-prone, and time-consuming part of the surgery is the insertion of the pedicle screw. The surgeon must determine the entry point and trajectory of the screw pilot holes from anterior-posterior and lateral fluoroscopic X-ray images, and not from axial ones. Clinical studies report 10\%-40\% misplaced screws, i.e., a screw that is more than $2 \mathrm{~mm}$ away from its ideal position , [5], [7], [8], [14], [17], [27]. About $3 \%$ of misplaced screws are more than $5 \mathrm{~mm}$ away from their planned position, causing nerve damage [8]. Fusion in the thoracic and cervical spine is even more risky because of the compact and delicate structure of the spine. Few experienced surgeons perform spinal fusion at these levels.

Pedicle screw insertion is well suited for computer-assisted surgery (CAS) techniques since it requires positioning a drill in a planned position with respect to the vertebra, which can be considered a rigid body. Most CAS solutions use a real-time tracking system to determine the relative position of the handheld drill and of the vertebra in real time. Some systems provide preoperative planning based on CT images [16], while others augment fluoroscopic X-ray images with a projection of the surgical tool at its current location [9]. While these systems allow the surgeon to place the drill at the desired position and orientation, they do not prevent the drill from skidding upon entrance and deviating as the drilling starts and proceeds. In addition, most current tracking systems are not minimally invasive, since they require acquiring sample points on the surface of the vertebra for registration purposes. An alternative approach is the use of custom-made templates [1], [19]. In this method, patient-tailored jigs are created from CT and fit to the spinous process during surgery to serve as a mechanical guide for the drill. While accurate, this technique cannot be applied in a minimally invasive approach. No robot-based systems have been routinely used for spinal surgery, since they would require real-time tracking of the vertebra to continuously adjust its position with respect to a fixed bed- or floor-mounted robot.

We are developing a system that uses the MARS robot to steadily and precisely position a targeting drill guide so that its guiding sleeve axis coincides with a predefined pedicle screw entry point and axis. The miniature robot is directly mounted on the spinous process of the vertebra onto which the pedicles

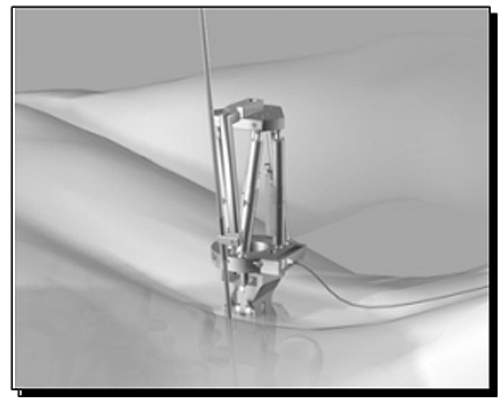

Fig. 5. The MARS robot in a spinal procedure. An axial view rendering with the robot mounted on the clamp, which is attached to the spinous process.

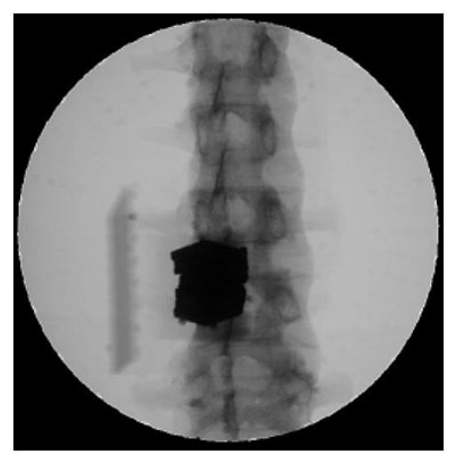

Fig. 6. Fluoroscopic X-ray image of the robot clamp attached to the vertebra.

screws are to be inserted (Fig. 5). With a couple of X-ray fluoroscopic images, the vertebra is registered to a CT-based preoperative plan on which the surgeon specifies the pedicle locations. The desired accuracy of the pedicle screw alignment is $\pm 1 \mathrm{~mm}$ along the pedicle axis. These tolerances guarantee that the screws can be inserted without excess deviation from the planned position and thus, will not cause vertebra perforation or nerve damage.

The surgical protocol and system operation is as follows. Preoperatively, a CT of the spine section, which will be operated on, is acquired and transferred to a planning computer. With the help of a graphical user interface (GUI) software, the surgeon determines the ideal positions and orientations of the left and right pedicles for each fused vertebra. The software can advise the surgeon on the safety of the screw position, orientation, and insertion depth with respect to the anatomical structures. The plan is then transferred to the operating room.

Intraoperatively, a small incision is made to expose the vertebra's spinous process. The robot is then attached to it with a clamp or with two K-wires. Next, a registration jig is attached to the robot, and several fluoroscopic X-ray images are taken (Fig. 6). The computer analyzes the images and determines the relative position of the robot base with respect to the vertebra it is attached to. The vertebra on the X-ray is matched to its CT counterpart using a model-based approach combining feature-based and intensity-based two-dimensional/three-dimensional (2-D/3-D) anatomic registration. X-ray distortion correction and calibration is performed with similar fluoroscopic images as described in [13]. Then, the registration jig is replaced with the targeting drill guide and the controller moves the robot so that the targeting guide's axis coincides with the 


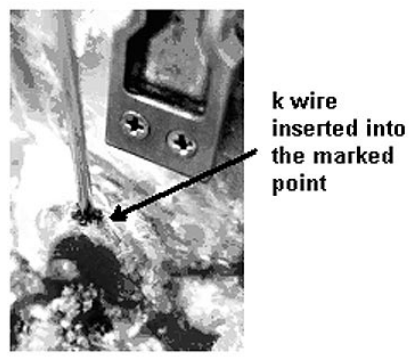

Fig. 7. Insertion of K-wires into pedicles in an animal test-marked sign around the K-wire is for placement error measurement.

planned pedicle screw axis. A K-wire is then inserted in the guide sleeve and a new fluoroscopic X-ray image is acquired to verify its correct positioning. Next, the surgeon inserts the drill into the guiding sleeve, drills the pilot hole, and inserts the pedicle screw. The procedure is repeated for the other pedicle and for the other vertebras. With the screws in place, the surgeon immobilizes the vertebra by inserting rigid connecting rods through pedicle screw heads. Although the rod insertion is more difficult that in the conventional approach because the incision is smaller and the pedicle screw heads are not exposed, it is still feasible without too much effort and skill. The RAS procedure is thus much less invasive than the conventional one.

We have built a working model of the miniature robot and have conducted preliminary tests on animals. We have successfully tested its attachment to the bone, determined that its workspace is adequate, and confirmed its mechanical ability to accurately guide a drill.

In a preliminary set of experiments, we studied the attachment of the robot to the spinous process with two K-wires [29]. We found that the expected deviation due to lateral forces and moments acting on the robot by the surgeon is less than $1 \mathrm{~mm}$ in the pedicle entry point and less than $1.5 \mathrm{~mm}$ in the intervertebral disk entry point. The experiments also show that the bone is sufficiently stiff to be considered fully rigid and that the deflection is greatly influenced by the clamp, the robot, and targeting guide materials. In subsequent cadaver tests, we attached a six-dimensional force-torque sensor to a mockup of the robot and asked a surgeon to drill holes with the targeting guide. Alignment errors were measured using postoperative CT scans. The measurement showed that drilling a pilot hole with the desired accuracy is possible, despite the lateral forces on the robot.

In the second set of experiments, we studied the rigidity of the robot and its attachment to the bone with a clamp on a pig spine (Figs. 7 and 8). We asked an experienced surgeon to manually mark the entry point on the pedicle. We then moved the robot with the drill guide to the predetermined point and recorded its position. We returned the robot to its home position, moved it back to the entry point, and asked the surgeon to drill a pilot hole applying a typical drilling load. We then measured the distance between the planned and the actual entry point to determine the positional error. Fig. 7 shows the inserted K-wire with the predrilled mark at the entry point. Table II quantifies the measurements of the K-wire entry point deviation. The experimental results analysis on several spinal levels on both sides of a pig spine (Table II) reveals a mean placement error of $0.2 \mathrm{~mm}$

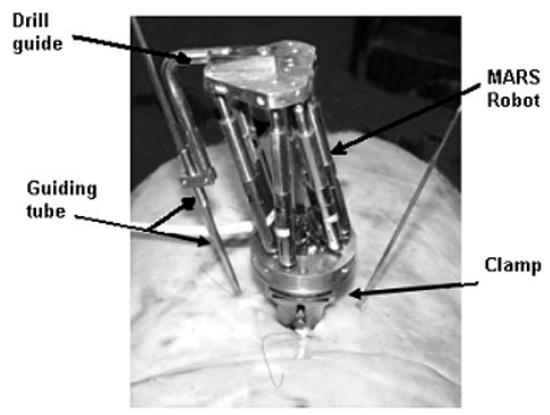

Fig. 8. Insertion of K-wire into pedicles in a minimally invasive approach.

TABLE II

EnTRY Point Positional ERror MEASUREMENT Diagram (LEFT) AND TyPiCAl MEAsurement VALUES From a K-Wire PlaCEMENT EXPERIMENT

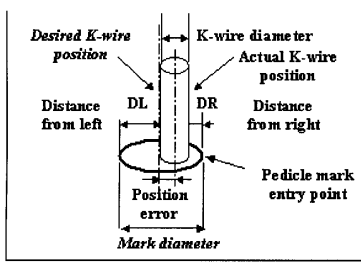

\begin{tabular}{|l|c|c|} 
Scaling factor & 5.88 & 1.00 \\
\hline Dimensions & Image $[\mathrm{mm}]$ & Actual [mm] \\
\hline Mark point diameter & 24.3 & 4.13 \\
\hline K-wire diameter & 11.76 & 2.00 \\
\hline Distance from the left - LD & 6.08 & 1.03 \\
\hline Distance from the right - RD & 7.12 & 1.21 \\
\hline K-wire + LD + RD & 24.96 & 4.24 \\
\hline Error placement left - LP & 0.19 & 0.03 \\
\hline Error placement right - RP & -0.85 & -0.14 \\
\hline Placement Error (max) & 0.85 & $\mathbf{0 . 1 4}$ \\
\hline
\end{tabular}

(maximum $0.48 \mathrm{~mm}$ ). Fig. 8 shows the percutaneous set up of K-wire insertion into the vertebras' pedicle.

\section{Distal LOCKING IN INTRAMEDULLARY NAILING}

The second clinical application of the bone-mounted miniature robot concept is distal locking in closed intramedullary nailing. Closed intramedullary nailing is currently the routine procedure of choice for reducing fractures of the femur and the tibia [4]. It restores the integrity of the fractured bone with a nail inserted in the medullary canal. The nail is inserted without surgically exposing the fracture through an opening, usually in the proximal bone. The surgeon reduces the fracture by manipulating the proximal and distal bone fragments through the leg until they are aligned. The surgeon then inserts a guide wire, reams the canal if necessary, and drives the nail in. In most cases, the surgeon inserts lateral proximal and distal interlocking screws to prevent fragment rotation and bone shortening.

Distal locking - the insertion of lateral screws to prevent nail rotation-has long been recognized as one of the most challenging steps in this procedure. Since the nail deforms by several millimeters to conform to the bone canal shape, the exact position of the distal locking nail holes' axes cannot be determined in advance. By repeatedly alternating between anterior-posterior and lateral X-ray fluoroscopic views, the surgeon adjusts the entry point and orientation of the drill so that its axis coincides with the corresponding nail hole axis (see Fig. 9). Drilling proceeds incrementally, with each advance verified with a new pair of X-ray fluoroscopic images. Once the pilot hole passing through the distal locking nail's hole has been drilled, the locking screw is fastened. Complications include inadequate fixation, malrotation, bone cracking, cortical wall penetration, and bone weakening due to multiple or enlarged 

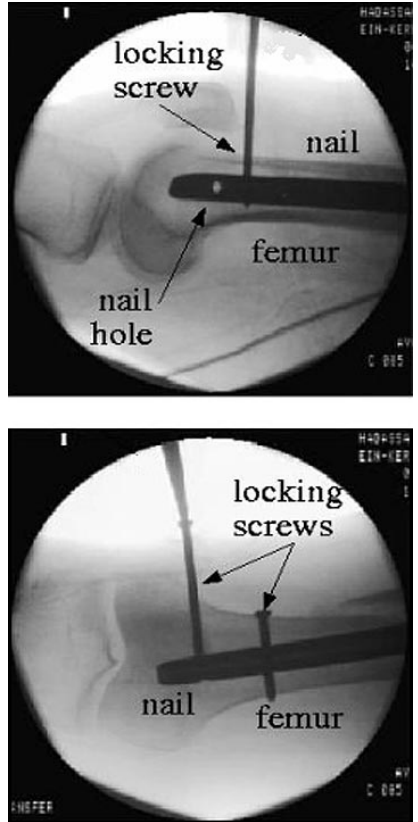

Fig. 9. X-ray fluoroscopic images taken during conventional distal locking. The lateral (top) view shows the distal part of the femur and the intramedullary nail with two distal locking nail holes. The frontal (bottom) view shows the intramedullary nail with two distal locking screws.

pilot holes. The literature reports that the surgeon's direct exposure to radiation per procedure is $3-30 \mathrm{~min}$, of which $31 \%-51 \%$ is spent on distal locking [23].

Many devices have been developed for distal locking [12]. Examples include proximally mounted targeting devices, stereo fluoroscopy, mechanical guides, and optical and electromagnetic navigation systems that help locate the center of the distal locking nail holes. However, all of these devices and techniques have deficiencies: they are only selectively applicable, are cumbersome and difficult to use, or are not sufficiently accurate, and thus fail to significantly reduce the likelihood of patient complications. Recent fluoroscopy-based navigation systems [9], [10], [11] take the guesswork out of targeting by tracking in real time the bone fragment position and augmenting static X-ray fluoroscopic images with projections of the surgical tools at their current location. The instruments' positions relative to the bone are continuously updated and viewed on screen as they move. With the help of these images, the surgeon aligns the drill axis with the distal locking nail hole axis to an accuracy of about 1 $\mathrm{mm}$ and $1^{\circ}$. However, since there is no mechanical guide for the handheld drill, it can slip or deviate from its planned trajectory as the drilling proceeds. Consequently, the surgical outcomes are still largely dependent on the surgeon's skill.

We are developing a system that uses the MARS robot to steadily and precisely position a targeting drill guide so that its guiding holes' axes (the surgical target) coincide with the distal locking nail holes' axes. The miniature robot is directly mounted laterally on the patient's bone segment distal to the fracture line and proximal to the distal locking nail holes, or on the proximal nail head via an extension mounting plate (Fig. 10). With just a few lateral fluoroscopic X-ray images, the targeting drill guide holes' axes and the distal locking nail holes' axes are brought into alignment by computing the transformation be-

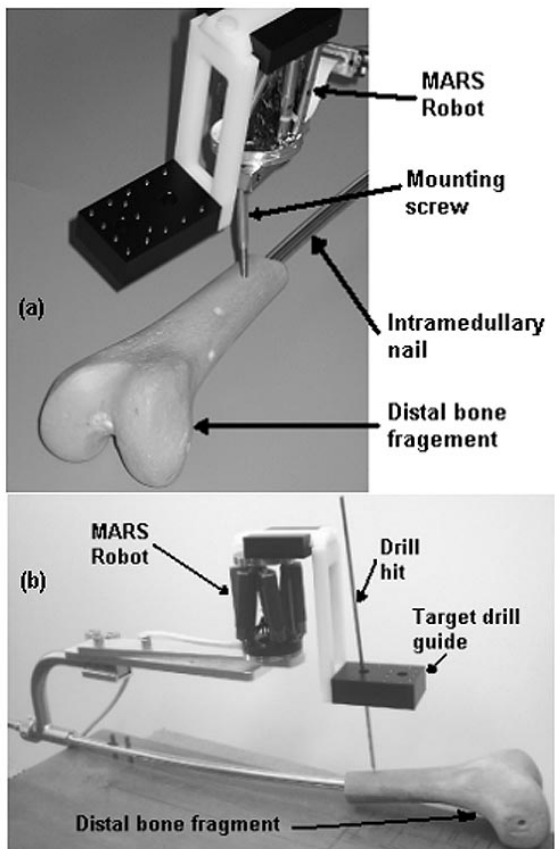

Fig. 10. Photograph of the MARS robot mounted on the femur (top) and on the nail head (bottom).

tween them and positioning the robot accordingly. The desired accuracy of the axis alignment is $\pm 1 \mathrm{~mm}$ of the screw entry point on the plane parallel to its axis, and axis angle deviation of $\pm 0.5^{\circ}$. These tolerances guarantee that the screws can be inserted without interference into the distal locking nail holes. The system was originally designed for the femur, but can be adapted with minor modifications to the tibia.

The surgical protocol and system operation is as follows. Once the fracture has been reduced and the nail has been inserted to its desired position, an image calibration ring is mounted on the fluoroscopic C-arm image intensifier. With a distal lateral fluoroscopic image showing the distal locking nail holes, the surgeon determines the location of the self-tapping screws on which the robot will be mounted. Their axes should be roughly parallel to the distal nail holes' axes and several centimeters proximal to them. The surgeon then drills, with the help of a handheld jig, two parallel pilot holes at $40-80 \mathrm{~mm}$ distance from them along the axis of the nail and $30 \mathrm{~mm}$ apart from each other. The self-tapping screws are then fastened and the targeting base that holds the robot base is mounted on it (Fig. 10). The targeting drill guide, which is made out of radiolucent Delrin, is then mounted on the robot top, and its position relative to the distal locking nail holes is roughly adjusted. The X-ray technician then adjusts the orientation of the $\mathrm{C}$-arm until the system determines that the distal locking nail holes appear as circles, as opposed to ellipses, in the image (a fronto-parallel view). This happens when the $\mathrm{C}$-arm imaging axis is parallel with the distal locking holes' axes.

The computer software then determines the relative position of the targeting drill guide with respect to these holes' axes and computes the transformation that will make the targeting drill guide holes' axes and the distal locking nail holes' axes coincide. The robot controller then moves the robot by the computed transformation and locks its links into position. The surgeon in- 


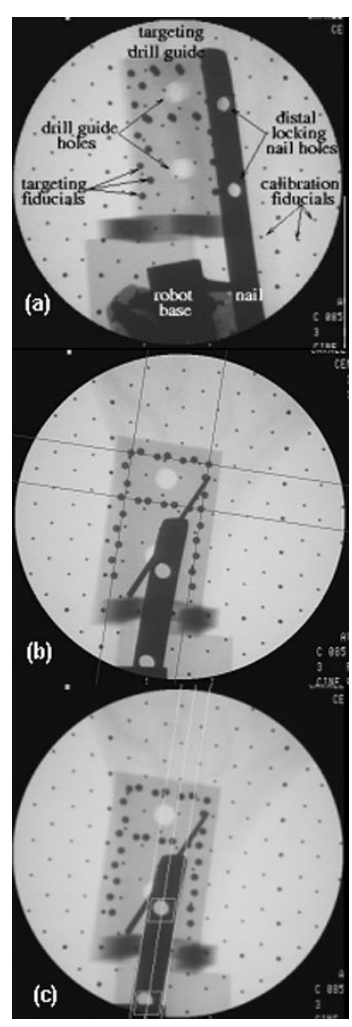

Fig. 11. X-ray fluoroscopic images showing: (a) the targeting drill guide and the distal end of the intramedullary nail with the two distal locking holes; (b) the identification of the targeting drill guide. The two orthogonal pairs of parallel lines are the targeting drill guide pattern; and (c) the identification of the distal locking nail holes. The parallel lines show the localization of the nail longitudinal contours. The two-parallelepiped windows show the localization of the hole.

serts a K-wire in each drill guide hole and verifies with a new pair of X-ray fluoroscopic images their alignment with respect to the distal locking nail hole centers. The surgeon proceeds to drill the pilot holes, removes the robot from its base and its screws, fastens the locking screws, and completes the surgery according to the standard protocol.

The goal of the registration is to align the targeting drill guide with the distal locking nail holes' axes. To localize the robot, we use a robot registration pattern that is incorporated into the targeting drill guide. It consists of $282-\mathrm{mm}$ spherical metal balls asymmetrically distributed on two parallel planes $20 \mathrm{~mm}$ apart. The balls form two orthogonal pairs of parallel lines [Fig. 11(a)]. The camera calibration and registration are performed with the same fluoroscopic X-ray images in four steps: 1) distortion correction and camera calibration [13]; 2) targeting drill guide localization [Fig. 11(b)]; 3) distal locking nail holes' axes localization [Fig. 11(c)]; and 4) registration. Many fiducials are used because a few fiducial occlusions due to overlap with other fiducials or other objects will always occur.

The targeting drill guide is localized by identifying its fiducials and the pattern they form. For individual fiducial identification, we use a Hough transform to detect the fiducial circles followed by normalized crosscorrelation to identify their centers. The major and minor axes of the targeting drill guide pattern are then determined from the fiducial locations using principal component analysis (PCA). The location of the distal locking nail holes is determined by first locating the nail's longitudinal contour with the Canny edge detector and then locating its holes from their expected position with respect to the contour. The search for the holes is confined to the strip defined by the nail contours and by sweeping in it a parallelepiped window whose sizes are equal to the nail width along the nail's medial axis.

The rigid transformation between the drill guide holes' axes and the distal locking nail holes' axes is computed as follows. Since the targeting drill guide is precalibrated, the transformation from the robot's coordinate system to targeting guide is known. The transformation between the targeting drill guide and the fluoroscopic $\mathrm{C}$-arm camera is determined from the extrinsic camera parameters and the known geometry of the targeting drill guide. To make the drill guide holes' axes and the distal locking nail holes' axes coincide, the system first orients the robot so that the drill guide holes' axes are aligned with the camera axis, and then translates until the targeting drill guide holes' and distal locking nail holes' center coincide.

\section{DISCUSSION AND CONCLUSION}

We have introduced the concept of a miniature bone-mounted robot to accurately position surgical tools and described its embodiment in MARS, a semiactive, bone-mounted miniature 6-DOF parallel robot. We are developing MARS-based systems for two clinical applications: 1) spinal pedicle screw insertion in spinal fusion and 2) distal locking in intramedullary femoral nailing. In both cases, the robot provides a mechanical guide for handheld screw pilot hole drilling based on planned positions of the screws' entry points and axes. The intraoperative registration between the planned screw positions and the anatomy is done with a few distortion-corrected X-ray fluoroscopic images. The additional procedure of X-ray imaging and mounting the robot on the bone is minimally invasive, with a small overhead that is offset by the benefits of precise mechanical positioning.

We have developed custom hardware and software for both applications, and are currently refining them and conducting in-vitro robustness and accuracy experiments. Our preliminary experiments show that the bone attachment is sufficiently rigid to withstand the lateral forces of handheld drill guiding and that the robot workspace is adequate for the task.

The main expected advantages of a bone-mounted miniature robot-based system over the existing conventional procedure are: 1) eliminate the trial-and-error process performed under $\mathrm{X}$-ray fluoroscopy required to position and orient the surgical tool; 2) provide a precise and steady mechanical guide to maintain the surgical tool in the right position and orientation during the operation; and 3) allow a minimally invasive approach. Since fewer X-ray views are necessary, the cumulative exposure of the surgeon and its staff to $\mathrm{X}$-ray radiation is also reduced. The system has the potential to reduce the occurrence of misplaced screws, to allow less skilled and less experienced surgeons to perform the surgery in the thoracic and cervical spine regions, and to shorten the surgery time.

The main expected advantages of the MARS robot-based system over other existing surgical robot and computer-aided 
navigation systems are: 1) the robot is much smaller, lightweight, and has a limited range of motions, so it is safer and easier to integrate in the operating room than larger robots; 2) mounting the robot directly on the bone eliminates the need for additional mechanical, optical, or electromagnetic hardware for bone motion tracking or patient immobilization; 3) the registration procedure between the robot and the planned surgical tool position and orientation is simpler and potentially more robust because the registration chain is shorter; 4) X-ray fluoroscopy can be used for planning and registration, eliminating the need for contact-based registration, which is currently the norm in commercial systems. Note that an essential requirement for both MARS-based and navigation-based systems is the rigid attachment of hardware (the robot or the dynamic reference frame) to the bone.

The MARS robot has been designed so it can be used in a variety of surgical procedures in which precise positioning and orientation of a handheld surgical tool in the vicinity of a rigid bony structure is required. Candidate procedures might include other orthopedic applications, such as bone cancer removal, various biopsies, and some neurosurgical, dental, maxillofacial, and ENT procedures. These possibilities are open for future exploration.

\section{REFERENCES}

[1] K. Birnbaum, E. Schkommodau, N. Decker, A. Prescher, U. Klapper, and K. Radermacher, "Computer-assisted orthopedic surgery with individual templates and comparison to conventional methods," Spine, vol. 26, pp. 365-370, 2001.

[2] C. Brack et al., "Accurate X-ray-based navigation in computer-assisted orthopaedic surgery," in Proc 12th Int. Symp. Computer-Assisted Orthopaedic Surgery, H. U. Lemke et al., Eds. New York: Springer, 1998.

[3] G. Brandt, K. Radermacher, S. Lavallee, H.-W. Staude, and G. Rau, "A compact robot for image guided orthopedic surgery: Concept and preliminary results," in Lecture Notes in Computer Science 1205, CVRMedMRCAS'97, J. Troccaz, R. Grimson, and R. Mosges, Eds. Berlin, Germany: Springer, 1997.

[4] R. J. Brumback, "Regular and special features-The rationales of interlocking nailing of the femur, tibia, and humerus," Clinical Orthopedics, Related Res., vol. 324, 1996.

[5] W. H. M. Castro, H. Halm, J. Jerosch, J. Malms, J. Steibeck, and S. Blasius, "Accuracy of pedicle screw placement in lumbar vertebrate," Spine, vol. 21, no. 11, pp. 1320-1326, 1996.

[6] K. Cleary and C. Nguyen, "State-of-the-art in surgical robotics: Clinical applications and technology challenges," Computer-Assisted Surgery, vol. 6 , no. 6, pp. 312-328, 2001 .

[7] H. Fahandezh-Saddi et al., "Complications associated with pedicle screw positioning," in Proc. 6th Int. Argos Symp., Paris, France, 2002.

[8] R. W. Gaines, "The use of pedicle screw internal fixation for spinal disorders," J. Joint and Bone Surgery, vol. 82-A, no. 10, pp. 1458-1489, 2000.

[9] R. Hofstetter, M. S. Slomczykowski, and L. P. Nolte, "Fluoroscopy as an imaging means for computer-assisted surgical navigation," ComputerAided Surgery, vol. 4, no. 2, pp. 65-76, 1999.

[10] L. Joskowicz et al., "Fluoroscopy-based navigation in computer-aided orthopaedic surgery," in Proc. IFAC Conf. Mechatronic Systems, Isermann et al., Eds., Darmstadt, Germany, 2000.

[11] L. Joskowicz, C. Milgrom, M. Shoham, Z. Yaniv, and A. Simkin, "Robot-guided long bone intramedullary distal locking: Concept and preliminary results," in Proc. 3rd Int. Symp. Robotics and Automation, ISRA'02, Toluca, Mexico, 2002.

[12] C. Krettek, B. Konemann, T. Miclau, R. Kolbli, T. Machreich, and H. Tscherne, "A mechanical distal aiming device for distal locking in femoral nails," Clinical Orthopedics, vol. 384, pp. 267-268, 1999.

[13] H. Livyatan, Z. Yaniv, and L. Joskowicz, "Robust automatic C-arm calibration for fluoroscopy-based navigation: A practical approach," in Proc. 5th Int. Conf. Medical Image Computing and Computer-Aided Intervention, Tokyo, Japan, 2002, pp. 60-68.
[14] S. C. Ludwig, D. L. Kramer, R. A. Balderstone, A. R. Vaccaro, K. F. Foley, and T. J. Albert, "Placement of pedicle screws in the human cadaveric cervical spine," Spine, vol. 25, no. 13, pp. 1655-1667, 2000.

[15] J. P. Merlet, Parallel Robots. Norwell, MA: Kluwer, 2000.

[16] L.-P. Nolte, H. Visarius, E. Arm, F. Langlotz, O. Schwarzenbach, and L. Zamorano, "Computer-aided fixation of spinal implants," $J$. Image-Guided Surgery (now Computer-Aided Surgery ), vol. 1, pp. 88-93, 1995.

[17] H. Pihlajamaki, "Complications of transpedicular lumbosacral fixation for nontraumatic disorders," J. Bone Joint Surgery, vol. 79-B, no. 2, pp. 183-189, 1997

[18] C. Riviere, R. S. Rader, and N. V. Thakor, "Adaptive cancelling of physiological tremor for improved precision in microsurgery," IEEE Trans. Biomed. Eng., vol. 45, pp. 839-846, July 1998.

[19] K. Radermacher, H. W. Staudte, and G. Rau, "CT-image-based planning and execution of interventions in orthopedic surgery using individual templates-Experimental results and aspects of clinical applications," in CAOS: Computer Assisted Orthopaedic Surgery, L.-P. Nolte and R. Ganz, Eds. Toronto, ON, Canada: Hogrefe \& Huber, 1998, pp. 1-11.

[20] Y. R. Rampersaud, K. T. Foley, A. C. Shen, S. Williams, and M. Solomito, "Radiation exposure to the spine surgeon during fluoroscopically assisted pedicle screw insertion," Eur. Spine J., vol. 25, no. 20, pp. 2637-2645, 2000.

[21] N. Sima'an, D. Glozman, and M. Shoham, "Design considerations of new types of six-degrees-of-freedom parallel manipulators," in Proc. IEEE Int. Conf. Robotics and Automation, vol. 2, May 1998, pp. 1327-1333.

[22] M. Shoham, M. Roffman, S. Goldberger, and N. Sima'an, "Robot construction for medical applications," in Proc. 2nd Israeli Symp. Computer Assisted Surgery, Medical Robotics and Medical Imaging, Jerusalem, Israel, 1999.

[23] S. Skejdal and S. Backe, "Interlocking medullary nails-Radiation doses in distal targeting," Archives of Orthopaedic Trauma Surgery, vol. 106, 1987.

[24] N. Sima' an and M. Shoham, "Robot construction for surgical applications," in Proc. 1st IFAC Conf. Mechatronics, 2000.

[25] M. Slomczykowski, M. Roberto, P. Schneeberger, C. Ozdoba, and P. Vock, "Radiation dose for pedicle screw insertion," Spine J., vol. 24, no. 10 , p. $975,1999$.

[26] R. H. Taylor, P. Jensen, L. Whitcomb, A. Barnes, R. Kumar, D. Stoianovici, P. Gupta, Z. Wang, E. deJuan, and L. Kavoussi, "A steady-hand robotic system for microsurgical augmentation," Int. J. Robot. Res., vol. 18, pp. 1201-1210, 1999.

[27] A. R. Vaccaro, S. J. Rizzolo, R. A. Balderstone, T. J. Allardyce, S. R. Garfin, C. Dolinskas, and H. S. An, "Placement of pedicle screws in the thoracic spine," J. Bone and Joint Surgery, vol. 77-A, no. 8, pp. 1193-1199, 1995.

[28] A. Wolf, M. Shoham, M. Schnider, and M. Roffman, "Morphometric study of the human lumbar spine for operation-workspace specifications," Spine J., vol. 26, no. 22, pp. 2472-2477, 2001.

[29] — - "Feasibility study of a mini robotic system for spinal operations: Analysis, and experiments," Eur. Spine J., 2003, to be published.

[30] L. W. Tsai, Robot Analysis: The Mechanics of Serial and Parallel Manipulators. New York: Wiley, 1999.

[31] Y. S. Yoon, J. J. Lee, D. S. Kwon, C. H. Won, A. J. Hodgson, and T. Oxland, "Accurate femoral canal shaping in total hip arthroplasty using a minirobot," in Proc. IEEE Int. Conf. Robotics and Automation, vol. 4, 2001, pp. 3214-3219.

[32] P. M. Tate, V. Lachin, L. Fu, H. Croitoru, and M. Sati, "Performance and robustness of automatic fluoroscopic image calibration in new computer-assisted surgery system," in Proc. MICCAI, 2001, pp. 1130-1136.

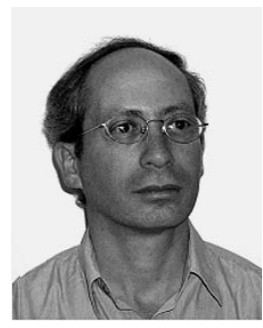

Moshe Shoham (A'89-M'95) has been conducting research in the robotics field for the past 20 years, with a special focus on kinematics and dynamics of robots, sensor integration, multifingered hands, and medical applications. He was heading the Robotics Laboratories, Mechanical Engineering Department, Columbia University, New York, 1986-1990, and is currently with the Technion-Israel Institute of Technology, Haifa, Israel. He co-chaired the past six Israeli symposiums on Computer-Assisted Surgery, Medical Robotics, and Medical Imaging.

Dr. Shoham has received several awards including the Haifa City Award for Science and Technology, the Mitchell Entrepreneurial Award, the Hershel Rich Innovation Award, and the Jolodan Prize. He served as an Associate Editor for the IEEE TRANSACTIONS ON RoBotics AND AUTOMATION from 2001 to 2003. 


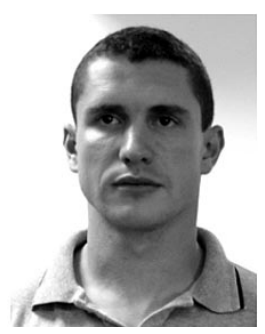

Michael Burman received the B.Sc. and M.Sc. degrees in mechanical engineering from the Technion-Israel Institute of Technology, Haifa, Israel, in 2000 and 2003, respectively.

$\mathrm{He}$ is the Mechanical Development Manager with Mazor Surgical Technologies, Caesarea, Israel, where he is responsible for the development of the MARS robot.

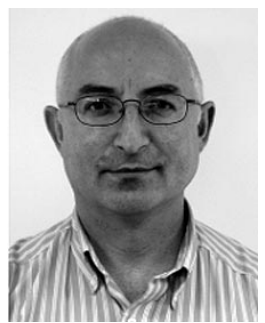

Eli Zehavi received the B.Sc. degree in electrical and computer engineering from Ben-Gurion University, Beer-Sheva, Israel, in 1984, and the M.B.A. degree in 2003.

$\mathrm{He}$ is currently responsible for developing and integrating the various elements that comprise products of Mazor Surgical Technologies, Caesarea, Israel. He was Director of Engineering at Elscint, Haifa, Israel, from 1996 to 2001 and as R\&D Project Department Manager at Gelman Sciences, Ann Arbor, MI, from 1988 to 1995 .

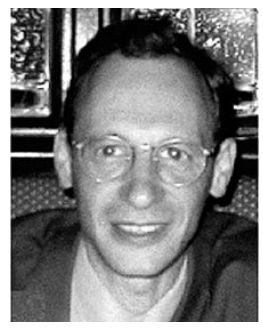

Leo Joskowicz (M'93-SM'95) received the Ph.D. degree in computer science from the Courant Institute of Mathematical Sciences, New York University, New York, NY, in 1988.

He is an Associate Professor at the School of Computer Science and Engineering at the Hebrew University of Jerusalem, Jerusalem, Israel, where he conducts research in computer-aided mechanical design, computer-assisted surgery, and robotics since 1995 . From 1988 to 1995, he was with the IBM T.J. Watson Research Center, Yorktown Heights, NY, where he conducted research in intelligent computer-aided design and computer-aided orthopaedic surgery. In 1996, he founded the Computer-Aided Surgery and Medical Image Processing Laboratory at the Hebrew University of Jerusalem. Since 2001, he has also been the Director of the Hebrew University's Leibniz Center for Research in Computer Science. He is a Member of the Editorial Boards of Computer-Aided Surgery, Medical Image Analysis, Artificial Intelligence in Engineering, and the Annals of Mathematics Artificial Intelligence. He has published extensively in these areas and has served on numerous related program committees.

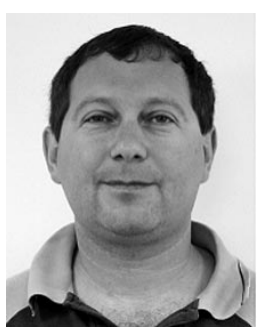

Eduard Batkilin received the Ph.D. degree in theoretical physics from the Technion-Israel Institute of Technology, Haifa, Israel, in 1995.

He is currently an Algorithm Development Manager with Mazor Surgical Technologies, Caesarea, Israel, where he is responsible for development of computer vision algorithms.

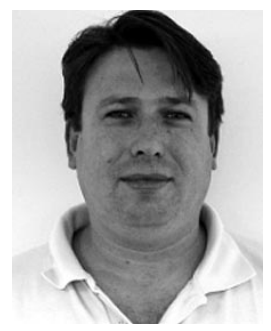

Yigal Kunicher received the B.Eng. degree (with honors) in electrical and electronic engineering from the University of Hertfordshire, Hatfield, U.K.

$\mathrm{He}$ is currently a System Engineer with Mazor Surgical Technologies, Caesarea, Isarael, and was previously an Electronics Supervisor with Elscint, Haifa, Israel from 1999 to 2001. 